\title{
Urothelial differentiation of human umbilical cord-derived mesenchymal stromal cells in vitro
}

\author{
Shuai $\mathrm{Wu}^{\mathrm{a}, \mathrm{b}, \mathrm{c}}$, Zhongliang Cheng ${ }^{\mathrm{a}, \mathrm{b}}$, Guohua Liu ${ }^{\mathrm{b}}$, Xinfeng Zhao ${ }^{\mathrm{d}}$, Liang Zhong ${ }^{\mathrm{b}}$, Yingjian Zhu ${ }^{\mathrm{b}, *}$ \\ and Jiang $\mathrm{Zhu}^{\mathrm{b}}$ \\ ${ }^{a}$ School of Medicine, Shanghai First People's Hospital, Shanghai Jiao Tong University, Shanghai, China \\ ${ }^{\mathrm{b}}$ Department of Urology, Shanghai First People's Hospital, Shanghai Jiao Tong University, Shanghai, China \\ ${ }^{\mathrm{c}}$ Department of Urology, Qingdao Municipal Hospital, Qingdao, China \\ ${ }^{\mathrm{d}}$ Section of Animal Models for Human Disease, Shanghai First People's Hospital, Shanghai Jiao Tong University, \\ Shanghai, China
}

\begin{abstract}
Human umbilical cord-derived mesenchymal stromal cells (hUCMSCs) are the most primitive of those isolated from other post-natal tissue source. The hUCMSCs possess the capability of differentiating along multi-lineage. This study aimed to investigate whether hUCMSCs can differentiate into urothelium-like cells. The hUCMSCs were isolated from fresh human umbilical cord postpartum and expanded at least to passage 3 in vitro. Subsequently, they were cultured with conditioned medium from urothelial cells (UC-CM) supplemented with $20 \mathrm{ng} / \mathrm{ml}$ exogenous epidermal growth factor (EGF). Urothelial cell specific marker uroplakin II (UPII) and cytokeratins were evaluated by reverse transcriptase-polymerase chain reaction (RT-PCR) and immunofluorescence technology. During culture, hUCMSCs started to express UPII and cytokeratins weakly at 7 days and were significantly up-regulated at 2 weeks post-induction. Additionally, morphology of hUCMSCs changed from spindle-shape to a polygonal epithelial-shape similar to that of urothelial cells after 7 days. The study results indicated that hUCMSCs can differentiate into urothelium-like cells in a defined micro-environment in vitro constituted by UC-CM and exogenous EGF.
\end{abstract}

Keywords: Cell differentiation, mesenchymal stromal cells, umbilical cord, urothelium

\section{Introduction}

Mesenchymal stem cells (MSCs) have immense therapeutic potential due to their capacity of selfrenewal and their ability to differentiate into multiple cell or tissue types. Over the last several years, intense research has been conducted to improve the characterization, isolation, and expansion of human MSCs [1-3]. The research findings have brought out the potential of these cells in the fields of therapeutics and tissue

\footnotetext{
*Corresponding author: Yingjian Zhu, Ph.D, MD, Department of Urology, Shanghai First People's Hospital, Shanghai Jiao Tong University, No 100, Haining Road, 200080 Shanghai, China. Tel.: +8621 63240090 3161; Fax: +86 2163241377 ; E-mail: zhuyingjian_sjtu@ 126.com.
}

engineering. These cells may be successfully collected and stored for both preclinical work and banking services $[4,5]$.

Bone-marrow represents one of the important sources for MSCs for both clinical and experimental purpose. However, bone marrow aspiration is a painful procedure and the frequency and differentiation potential of MSCs decrease significantly with age [6, 7]. Currently, umbilical cord (UC) is gaining attention for its therapeutic potential and as an accessible source of MSCs which can be easily processed after birth. Thus, UC can be considered as an alternative source of MSCs. Previous studies have demonstrated that human umbilical cord-derived mesenchymal stromal cells (hUCMSCs) are the most primitive MSCs among 
those isolated from other post-natal tissue sources and do not express the major histocompatibility complex (MHC) class II (HLA-DR) [8]. The UCMSCs show low immunogenicity, following xenotransplantation of human or pig UCMSCs into immune-competent rats $[9,10]$; lower than the other MSCs isolated from bone marrow and fat tissue [8]. Apart from their prominent advantages like abundant availability, painless collection, and faster self-renewal, hUCMSCs possess the capability to differentiate into a variety of cells of three germ layers in vitro, such as muscle cells [11], hepatocyte cells [12], and endothelial cells [13] which includes bone, cartilage, adipose cells, neural cells $[14,15]$.

Recent studies have demonstrated that hUCMSCs do not form any teratomas when injected into severe combined immunodeficiency (SCID) mice [16, 17]. In light of these circumstances, we speculated that hUCMSCs could have the potential to differentiate into urothelium-like cells and might be an ideal alternative cell source for bladder tissue engineering. According to previous studies $[18,19]$, epidermal growth factor (EGF) may be a critical element for differentiation of MSCs into urothelium-like cells.

In the current study, we hypothesized that conditioned medium from urothelial cells (UC$\mathrm{CM}$ ), supplemented with exogenous EGF would be more functional in differentiating hUCMSCs into urothelium-like cells, which expresses urothelial cell specific marker uroplakin II (UPII) and cytokeratins. Therefore, this study was conducted with the objective to describe the methodology of differentiating hUCMSCs into urothelium-like cells and to assess uroplakin II (UPII) and cytokeratins followed by cell differentiation.

\section{Material and methods}

\subsection{Isolation and culture of hUCMSCs}

With a proper written consent from parents, human umbilical cords were aseptically obtained from fullterm cesarean-section infants at Shanghai Jiao Tong University, affiliated to First People's Hospital. The use of this material was approved by the institutional review board of Chinese Academy of Medical Science and Medical School of Shanghai Jiao Tong University. Umbilical cords were obtained from 10 healthy women (age $<35$ years old) and stored aseptically in cold
Dulbecco's modified Eagle medium (DMEM). Cellular isolation started within 4 hours postpartum. The hUCMSCs isolation was performed as described previously [20-22]. The blood cells from arteries and veins were removed by flushing phosphate buffered saline (PBS) through the vessels and the cord was pulverized into approximately $1-2 \mathrm{~mm}^{3}$ large pieces. The cord pieces were transferred to cell culture flasks and incubated in DMEM with low glucose (DMEMLG, Invitrogen-Gibco, Carlsbad, CA) supplemented with $10 \%$ fetal bovine serum (FBS, Invitrogen-Gibco, Carlsbad, CA) at $37^{\circ} \mathrm{C}$ in a humidified atmosphere with $5 \% \mathrm{CO}_{2}$. The medium was changed every two days after initial plating. At the beginning, outgrowth of an adherent cell layer from single cord pieces was observed approximately after 10 days. After 2 weeks, tissue pieces were removed and adherent cells were harvested with $0.25 \%$ trypsin (Invitrogen-Gibco, Carlsbad, CA) treatment. The obtained cell suspension was centrifuged at $200 \mathrm{~g}$ for 5 minutes and the cells were resuspended in DMEM-LG supplemented with $10 \%$ FBS and subcultured at a density of $1 \times 10^{4}$ cells $/ \mathrm{cm}^{2}$. Only cells from passage 3 to 6 were used for subsequent studies. In this study, we performed flow cytometry analysis of cultured hUCMSCs. Our data showed hUCMSCs were positive for MSC markers, such as CD44, CD73, CD90, CD105, and negative for CD14, CD31, CD34 and CD45.

\subsection{Urothelial differentiation of hUCMSCs in vitro}

Urothelial cells used in this study were obtained from a commercial source (SV-HUC-1, Shanghai Institutes for Biological Sciences, Yueyang road 320. Shanghai, China) and cultured with Ham's F-12K medium (Invitrogen, Carlsbad, CA) supplemented with $4 \%$ FBS. Conditioned medium (CM) was derived from urothelial cells by collecting the media from cultured urothelial cells at 70-90\% confluence every 48 hours. Collected CM was centrifuged at $300 \mathrm{~g}$ for 5 minutes and filtered through a $0.22 \mu \mathrm{m}$ filter to remove cells and microorganisms, respectively. The UC-CM was prepared by diluting the collected CM with equal volume of DMEM-LG with FBS at a final concentration of $2 \%$. For urothelial differentiation, three experimental groups of hUCMSCs were established and cultured initially at 2000-4000 cells $/ \mathrm{cm}^{2}$ in DMED-LG with $10 \%$ FBS for 48 hours. After 2 days, media from three groups were replaced with 
UC-CM only, UC-CM $+20 \mathrm{ng} / \mathrm{ml}$ EGF (ProSpec, Rehovot Science Park, POB 398), or $30 \mathrm{ng} / \mathrm{ml}$ EGF + DMED-LG, respectively. The medium was changed every two days after 48 hours of initiation and cells were passaged by digestive method $[0.25 \%$ trypsin and $1 \mathrm{mM}$ ethylenediamine tetraacetic acid (EDTA)] at 80\%-90\% confluence. To assess the possibility of differentiation of cells into urothelium-like cells, hUCMSCs in three groups were individually analyzed on Days 0,7 and 14 with reverse transcriptase-polymerase chain reaction (RT-PCR) and immunofluorescence technology.

To understand the necessity of UC-CM and EGF for directing hUCMSCs differentiation to urothelium-like cells, we designed three exprimental groups: UC-CM, UC-CM + EGF, DMEM + EGF. The urothelial cells were used as positive control group.

\subsection{RNA extraction and RT-PCR}

Total cellular RNA was extracted from UC-induced hUCMSCs at day 0,7 and 14 using Trizol reagent (Invitrogen, Carlsbad, CA) which subsequently had been reversely transcribed into complementary DNA using SuperScript ${ }^{\mathrm{TM}}$ Reverse-Transcriptase reagents (Invitrogen, Carlsbad, CA) according to the manufacturer's instructions. Human glyceraldehyde 3-phosphate dehydrogenase (GAPDH) primers were used as an internal standard. Finally, PCR products were separated by $2 \%$ agarose gel-electrophoresis and observed under UV illumination.

The primers sequence was as follows:

Human cytokeratin 18:

Sense: CCGTCTTGCTGCTGATGA

Antisense: TTCCTCTTCGTGGTTCTTCTT

Human UPII

Sense: CCGCAAGTAAGGAGGTCTG

Antisense: GGAATAATGGAGTGTTGGAAGG

Human GAPDH

Sense: CAGTCAGCCGCATCTTCTT

Antisense: TGAGTCCTTCCACGATACCA

\subsection{Immunochemistry}

Cells were fixed with $4 \%$ paraformaldehyde for 20 minutes at room temperature, permeabilized with $0.25 \%$ triton X-100 (Sigma, USA) in PBS and blocked with $0.1 \%$ bovine serum albumin (BSA). Subsequently, cells were incubated overnight at $4^{\circ} \mathrm{C}$ with the following primary antibodies: anti-uroplakin II (UPII) (Santa Cruz, 1:50) [catalogue number: sc-15179] and anti-cytokeratins (Dako, 1:200) [catalogue number: M3515]. Although cytokeratins is not a specific marker for urothelial cells, we used it in combination with another specific urothelial cell marker UPII [19]. After washing three times with PBS, the cells were reacted with the appropriate fluorescence conjugated secondary antibody Alexa Fluor 555 and Alexa Fluor 488 (Invitrogen, diluted at 1:400) for 30 minutes at $37^{\circ} \mathrm{C}$. Finally, the cells were rinsed twice with PBS and counterstained with 4', 6-diamidino-2-phenylindole, dihydrochloride (DAPI, Invitrogen, 1:500) and then visualized by fluorescence microscopy (Nikon Eclipse E600).

\section{Results}

\subsection{Characterization of hUCMSCs}

Before differentiation, hUCMSCs exhibited predominantly spindle-shaped morphology, a characteristic of monolayer growth pattern (Fig. 1A). After 14 days of induction culture, cells were changed from spindle-shape to a polygonal epithelium-shape; similar to that of urothelial cells by morphologic observation when cultured with UC-CM + EGF (Fig. 1B). Urothelial cells were used as positive control (Fig. 1C).

\subsection{Induced hUCMSCs expressed urothelial-specific genes analyzed by RT-PCR}

To assess the possibility of urothelial differentiation, hUCMSCs were evaluated by RT-PCR with urothelialspecific genes. After 7 days of culture, the results showed that hUCMSCs with UC-CM + EGF expressed cytokeratin18 (CK18) and UPII mRNA. Moreover, those mRNA were expressed markedly after 14 days of culture (Fig. 2), which was similar to those of UC (positive control). However, both CK18 and UPII mRNA were completely absent in hUCMSCs (negative control), cultured with UC-CM only, or DMEM + EGF (data not shown).

\subsection{Expression of urothelial markers in induced hUCMSCs analyzed by immunofluorescence staining}

To confirm the results of differentiation, immunofluorescence studies were performed on all the three 

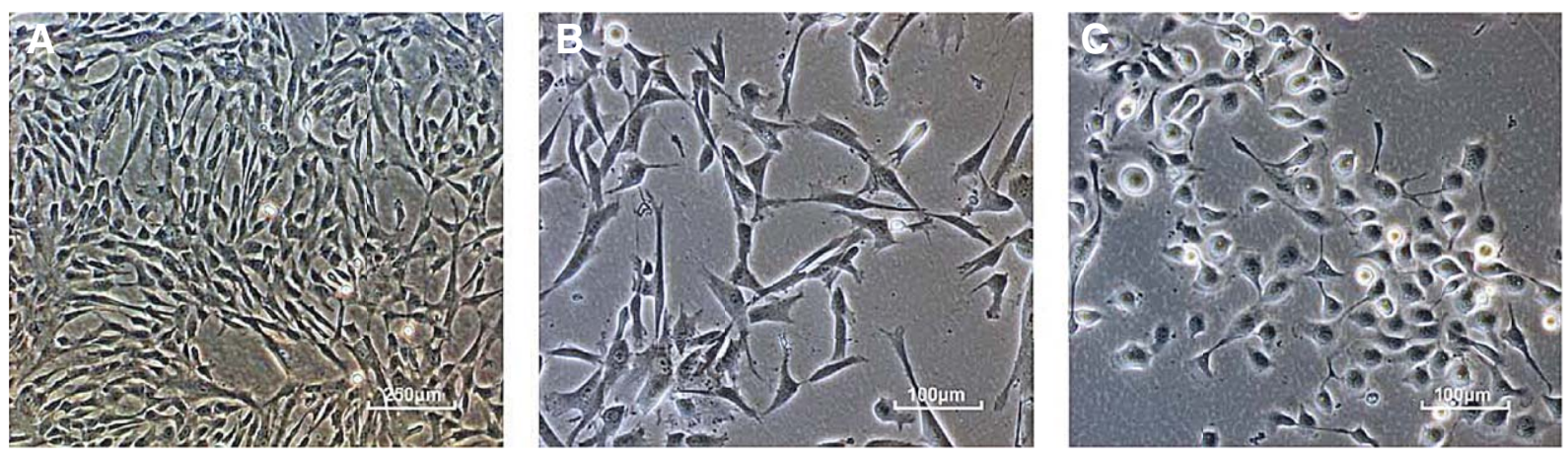

Fig. 1. Light microscopy of hUCMSCs and UC. A. hUCMSCs appear spindle-shaped morphology characterized of mono-layer growth pattern. B. Induced hUCMSCs exhibit polygonal epithelium-shape similar to UCs after 2 weeks induction with UC-CM supplemented with $20 \mathrm{ng} / \mathrm{ml}$ EGF. C. UCs exhibit typical cobblestone morphology. hUCMSCs: human umbilical cord-derived mesenchymal stromal cells. UCs: urothelial cells. UC-CM: conditioned medium derived from urothelial cells. EGF: epidermal growth factor.

groups. In the present study, approximately $40 \%-60 \%$ hUCMSCs in UC-CM + EGF group expressed UPII protein, a specific antigen for urothelial cells, and cytokeratins at Day 14. The expression of these markers was stronger after 2 weeks of culture than at 1 week. However, neither UPII nor cytokeratins were expressed in other groups during 2 weeks of culture. Urothelial cells were used as positive control (Fig. 3).

Immunochemical and RT-PCR analysis showed UPII positive cells only in group UC-CM + EGF after 2 weeks of induction. In contrast, both UC-CM and EGF alone failed to induce urothelial differentiation of hUCMSCs. Therefore, both UC-CM and EGF were necessary for urothelial differentiation. However, flow cytometry analysis of induced hUCMSCs was helpful to compare the yield and purity of UPII positive cells among different groups.

\section{Discussion}

Most previous studies have reported that cells and their secreted proteins constitute a functional niche in vivo, which orchestrates the balance of self-renewal and differentiation in all stem cells [23, 24]. Similarly, all functional elements in the conditioned medium including various kinds of proteins and ions may determine the fate of stem cells. Our present data demonstrated that provision of an appropriate microenvironment from UC-CM with extra exogenous EGF can direct and promote the differentiation of hUCMSCs toward urothelium. In this study, we initially cultured hUCMSCs with UC-CM alone for two weeks and found no expression of UPII and cytokeratins

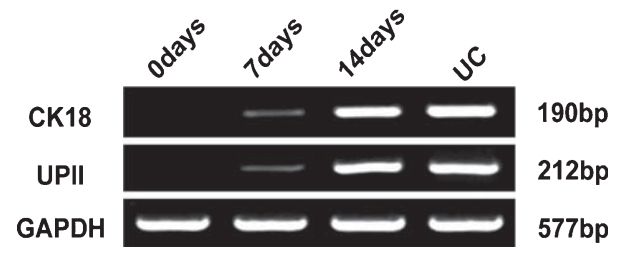

Fig. 2. hUCMSCs were analyzed by RT-PCR after urothelial differentiation. hUCMSCs were cultured with UC-CM supplemented $20 \mathrm{ng} / \mathrm{ml}$ exogenous EGF. After differentiation, hUCMSCs were analyzed by RT-PCR with uroplakin II (UP II) and cytokeratin 18 (CK18). UCs were used as positive control. GAPDH primers were used as an internal standard.

detected by RT-PCR and immunofluorescence technology. Using the method from a previous study [19] as reference, we have tried to induce hUCMSCs differentiation into urothelium by DMEM supplemented with $30 \mathrm{ng} / \mathrm{ml}$ EGF and 2\% FBS. After two weeks of differentiation, expression of urothelial marker UPII, or cytokeratins was not observed (data not shown). UP Ia/UP II and UP Ib/UP III can be observed in the intermediate and terminal course of urothelial cell differentiation respectively. The urothelial differentiation of stem cells can hardly reach the terminal stage in vitro and seldom have enough UP Ib/UP III that is detectable. Hence, we selected one of these urothelial specific markers (UP II) for estimation. Liu et al. also reported that uroplakin Ib and cytokeratin 18 were observed in differentiated hASCs by immunofluorescence [18].

We have speculated that the defined inductive medium incorporating UC-CM with exogenous EGF may be more functional to promote hUCMSCs' differentiation. In order to determine this, the present 

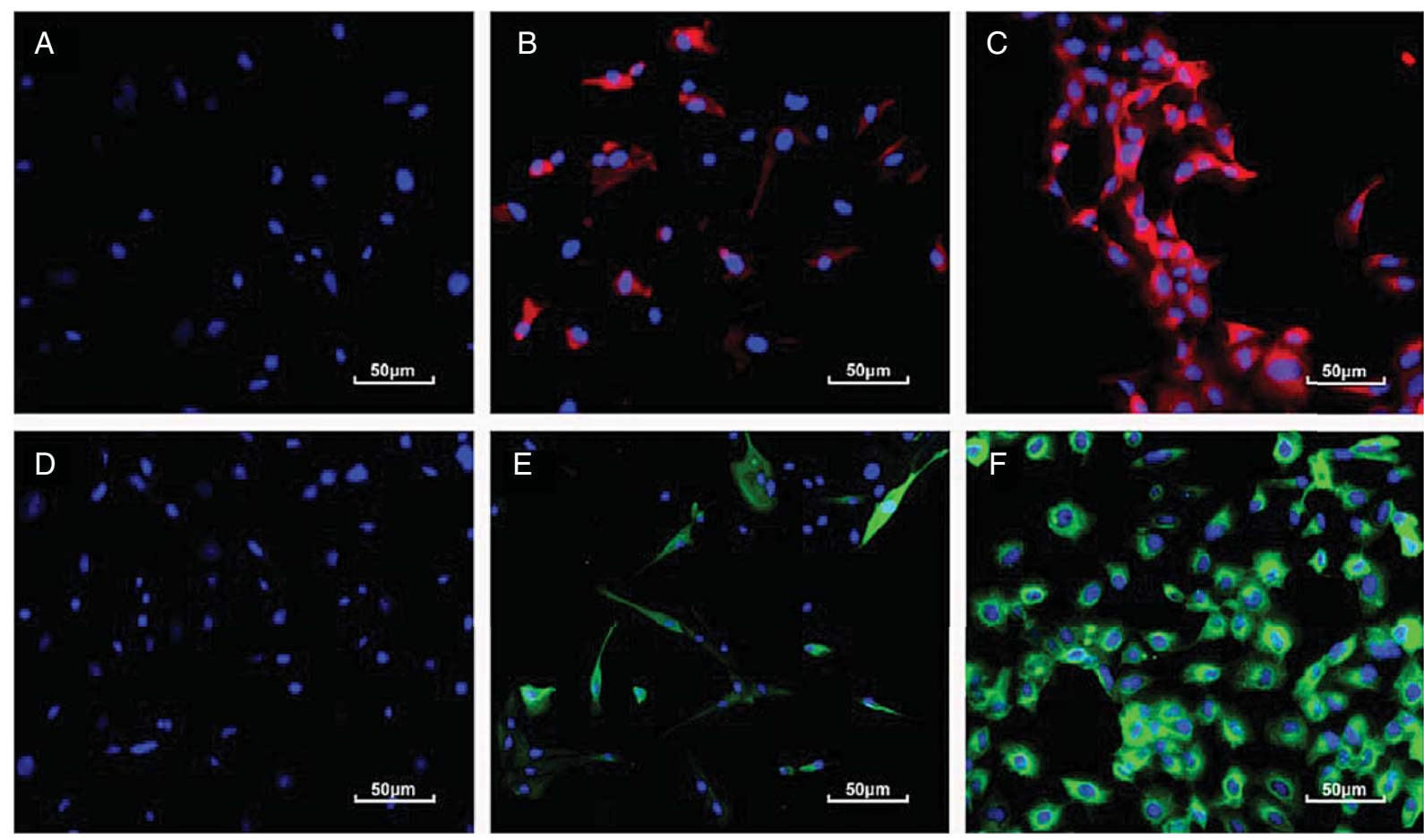

Fig. 3. hUCMSCs were assessed by immunofluorescence staining after differentiation. hUCMSCs cultured with UC-CM supplemented 20 ng/ml exogenous EGF were assessed by immunofluorescence staining at day 0 (A, D), days 14 (B, E). UCs were used as positive control (C, F). Cells were immunostained for cytokeratins (red) (A, B, C) and uroplakin II (green) (D, E, F).

experiment was performed to differentiate hUCMSCs into urothelium-like cells by UC-CM supplemented with exogenous EGF. In this study, we have tried to use different concentrations of exogenous EGF for differentiation of hUCMSCs, such as $5 \mathrm{ng} / \mathrm{ml}, 10 \mathrm{ng} / \mathrm{ml}$, $15 \mathrm{ng} / \mathrm{ml}, 20 \mathrm{ng} / \mathrm{ml}$ and so on. Finally, we have succeeded in directing the differentiation of hUCMSCs toward urothelium-like cells by UC-CM supplemented with $20 \mathrm{ng} / \mathrm{ml}$ exogenous EGF.

By contrast, we have found that EGF alone was insufficient to induce the differentiation of hUCMSCs into urothelium. This difference might be related to the cell types. In the previous study [19], human urine-derived stem cells were more homoplastic to urothelium. Their fate may be already half-directed on account of their origination and were prone to be induced with EGF only. However, hUCMSCs are the most primitive and need some active elements to direct differentiation of cells into urothelium. The UC-CM could play a critical role in directing differentiation of hUCMSCs into urothelium-like cells. A study conducted by Shi et al. [25], reported that human adipose-derived stem cells (HADSCs) showed in vitro upregulation of markers for differentiation towards urothelial cells by culturing in an urothelialconditioned medium, which provides an alternative cell source for potential use in urinary tract tissue engineering.

However, we could not succeed in differentiating cells toward urothelium by UC-CM alone. In the study by $\mathrm{Wu}$ et al. [19], EGF was found as a critical element for differentiation of cells into urothelium-like cells. With this in mind, we added some exogenous EGF in UC-CM. Ning et al. [26] conducted a study to determine the ability of cultured bone marrow-derived mesenchymal stem cells (BMSCs) to differentiate into functional urothelium. They concluded that co-cultured BMSCs had microstructural features characteristic of epithelial cells. We have also succeeded in orienting and promoting hUCMSCs differentiation into urothelium-like cells by UC-CM supplemented with $20 \mathrm{ng} / \mathrm{ml}$ exogenous EGF, which expressed UPII and cytokeratins on the level of genes and proteins. The results presented here indicated 
that UC-CM might provide an urothelial direction and partial power for differentiation of hUCMSCs. The exogenous EGF might further assist the differentiation of hUCMSCs into urothelium.

\section{Conclusions}

In summary, we have shown that hUCMSCs possess a capability of differentiating into urothelium-like cells in an appropriate micro-environment in vitro. The UC-CM and EGF can constitute a functional niche to promote differentiation of hUCMSCs. However, further work is required to identify whether hUCMSCs undergo complete differentiation toward urothelium in vivo after implantation into human or animal bodies.

\section{Acknowledgments}

This study is supported by grants from the Research Program of Science and Technology Commission of Shanghai Municipality (10411967200) and Shanghai Song-Jiang Health Bureau (2011PD06) and National Natural Science Foundation of China (81170642) and Shanghai Shen Kang plat-form Grant (SHDC12007206).

\section{Conflict of interest}

There is no conflict of interest among the authors.

\section{References}

[1] M. Secco, E. Zucconi, Vieira, et al. Mesenchymal stem cells from umbilical cord: Do not discard the cord, Neuromuscul Disord 18 (2008), 17-18.

[2] D.L. Troyer and M.L. Weiss, Wharton's jelly-derived cells are a primitive stromal cell population, Stem Cells 26 (2008), 591-599.

[3] R. Sarugaser, J. Ennis, W.L. Stanford and J.E. Davies, Isolation, propagation, and chaarcterization of human umbilical cord perivascular cells (HUCPVCs), Methods Mol Biol 482 (2009), 269-279.

[4] S. Kestendjieva, D. Kyurkchiev, G. Tsvetkova, T. Mehandjiev, A. Dimitrov, A. Nikolov and S. Kyurkchiev, Characterization of mesenchymal stem cells isolated from the human umbilical cord, Cell Bio Int 32 (2008), 724-732.

[5] K. Seshareddy, D. Troyer and M.L. Weiss, Method to Isolate Mesenchymal-Like Cells from Wharton's Jelly of Umbilical Cord, Methods Cell Biol 86 (2008), 101-119.
[6] V.D. Roobrouck, F. Ulloa-Montoya and C.M. Verfaillie, Selfrenewal and differentiation capacity of young and aged stem cells, Exp Cell Res 314 (2008), 1937-1944.

[7] K. Stenderup, J. Justuesen, C. Clausen and M. Kassem, Aging is associated with decreased maximal life span and accelerated senescence of bone marrow stromal cells, Bone 33 (2003), 919-926.

[8] M.L. Weiss, C. Anderson, S. Medicetty, et al. Immune Properties of Human Umbilical Cord Wharton's Jelly-Derived Cells, Stem Cells 26 (2008), 2865-2874.

[9] M.L. Weiss, S. Medicetty, Bledsoe et al. Human umbilical cord matrix stem cells: Preliminary characterization and effect of transplantation in a rodent model of Parkinson's disease, Stem Cells 24 (2006), 781-792.

[10] S. Medicetty, A.R. Bledsoe, C.B. Fahrenholtz, D. Troyer and M.L. Weiss, Transplantation of pig stem cells into rat brain: Proliferation during the first 8 weeks, Exp Neurol 190 (2004), 32-41.

[11] M.T. Conconi, P. Burra, et al. CD105(+) cells from Wharton's jelly show in vitro and in vivo myogenic differentiative potential, Int J Mol Med 18 (2006), 1089-1096.

[12] D. Campard, P.A. Lysy, M. Najimi and E.M. Sokal, Native umbilical cord matrix stem cells express hepatic markers and differentiate into hepatocyte-like cells, Gastroenterology 134 (2008), 833-848.

[13] K.H. Wu, B. Zhou, S.H. Lu, et al. In vitro and in vivo differentiation of human umbilical cord derived stem cells into endothelial cells, J Cell Biochem 100 (2007), 608-616.

[14] Y.S. Fu, Y.T. Shih, Y.C. Cheng and M.Y. Min, Transformation of human umbilical mesenchymal cells into neurons in vitro, J Biomed Sci 11 (2004), 652-660.

[15] K.E. Mitchell, M.L. Weiss, B.M. Mitchell, P. Martin, D. Davis, L. Morales, B. Helwig, M. Beerenstrauch, K. Abou-Easa, T. Hildreth, D. Troyer and S. Medicetty, Matrix cells from Wharton's jelly form neurons and glia, Stem Cells 21 (2003), 50-60.

[16] R. Sodian, C. Lueders, L. Kraemer, et al. Tissue engineering of autologous human heart valves using cryopreserved vascular umbilical cord cells, Annals of Thorac Surg 81 (2006), 2207-2216.

[17] R.S. Rachakatla, F. Marini, M.L. Weiss, M. Tamura and D. Troyer, Development of human umbilical cord matrix stem cell-based gene therapy for experimental lung tumors, Cancer Gene Ther 14 (2007), 828-835.

[18] J. Liu, J. Huang, T. Lin, C. Zhang and X. Yin, Cell-to-cell contact induces human adipose tissue-derived stromal cells to differentiate into urothelium-like cells in vitro, Biochem Biophys Res Commun 390 (2009), 931-936.

[19] S. Wu, Y. Liu, S. Bharadwaj, A. Atala and Y. Zhang, Human urine-derived stem cells seeded in a modified $3 \mathrm{D}$ porous small intestinal submucosa scaffold for urethral tissue engineering, Biomaterials 32 (2011), 1317-1326.

[20] G. La Rocca, R. Anzalone, S. Corrao, F. Magno, T. Loria, M. Lo Iacono, A. Di Stefano, P. Giannuzzi, L. Marasa, F. Cappello, G. Zummo and F. Farina, Isolation and characterization of Oct-4+/HLA-G + mesenchymal stem cells from human umbilical cord matrix: Differentiation potential and detection of new markers, Histochem Cell Biol 131 (2009), 267-282.

[21] L.L. Lu, Y.J. Liu, S.G. Yang, Q.J. Zhao, X. Wang, W. Gong, Z.B. Han, Z.S. Xu, Y.X. Lu, D. Liu, Z.Z. Chen and Z.C. Han, Isolation and characterization of human umbilical cord 
mesenchymal stem cells with hematopoiesis-supportive function and other potentials, Haematologica 91 (2006), 10171026.

[22] I. Majore, P. Moretti, R. Hass and C. Kasper, Identification of subpopulations in mesenchymal stem cell-like cultures from human umbilical cord, Cell Commun Signal, 7-6.

[23] E. Fuchs, T. Tumbar and G. Guasch, Socializing with the neighbors: Stem cells and their niche, Cell 116 (2004), 769-778.

[24] K.A. Moore and I.R. Lemischka, Stem cells and their niches, Science 311 (2006), 1880-1885.
[25] J.G. Shi, W.J. Fu, X.X. Wang, Y.D. Xu, G. Li, B.F. Hong, K. Hu, F.Z. Cui, Y. Wang and X. Zhang, Transdifferentiation of human adipose-derived stem cells into urothelial cells: Potential for urinary tract tissue engineering, Cell Tissue Res 347(3) (2012), 737-746.

[26] J. Ning, C. Li, H. Li and J. Chang, Bone marrow mesenchymal stem cells differentiate into urothelial cells and the implications for reconstructing urinary bladder mucosa, Cytotechnology 63 (2011), 531-539. 


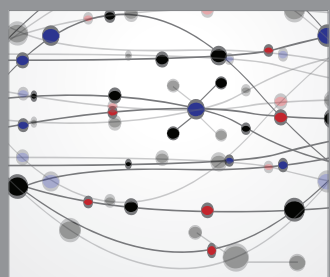

The Scientific World Journal
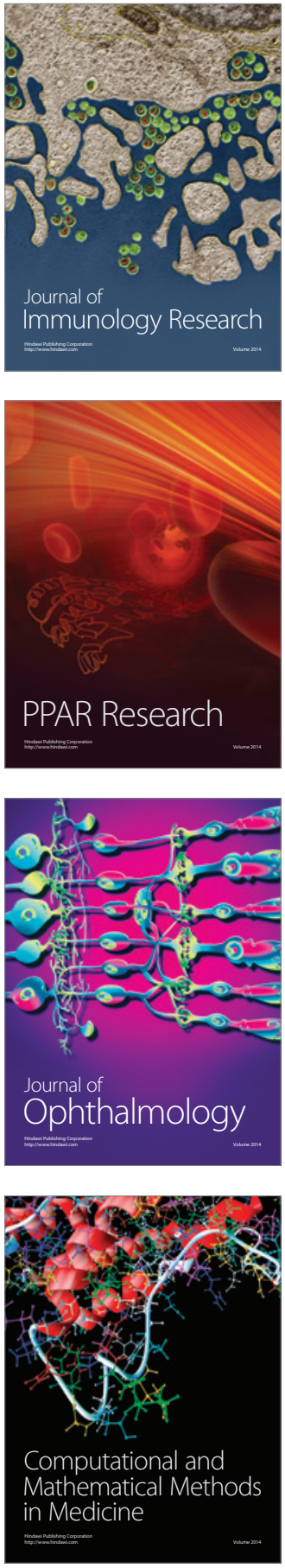

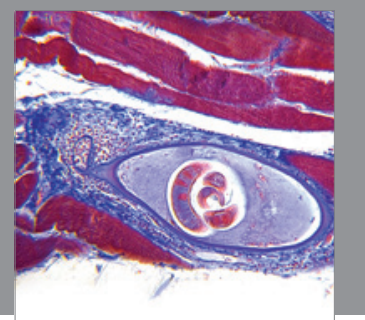

Gastroenterology

Research and Practice
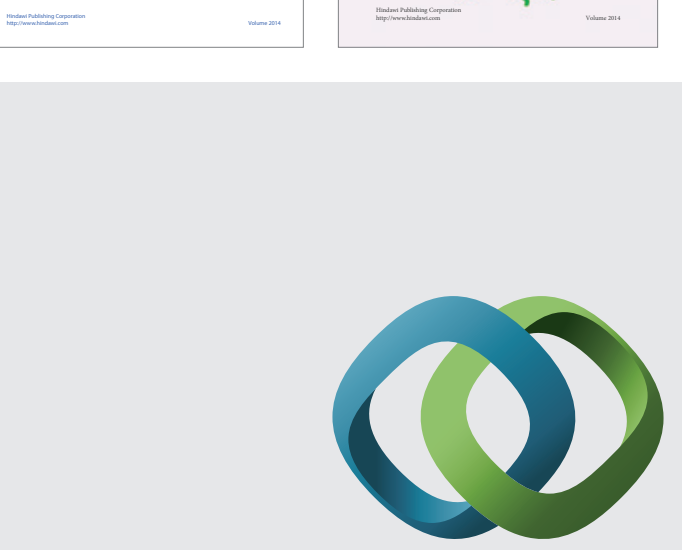

\section{Hindawi}

Submit your manuscripts at

http://www.hindawi.com
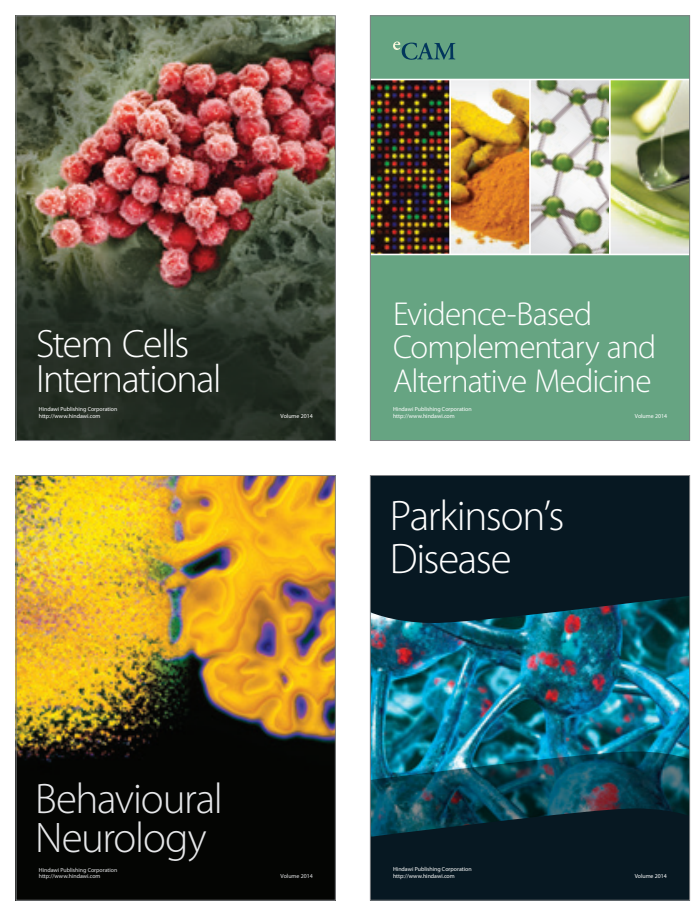

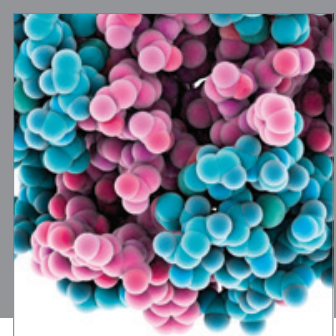

Journal of
Diabetes Research

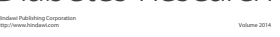

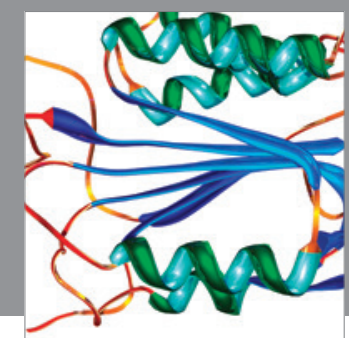

Disease Markers
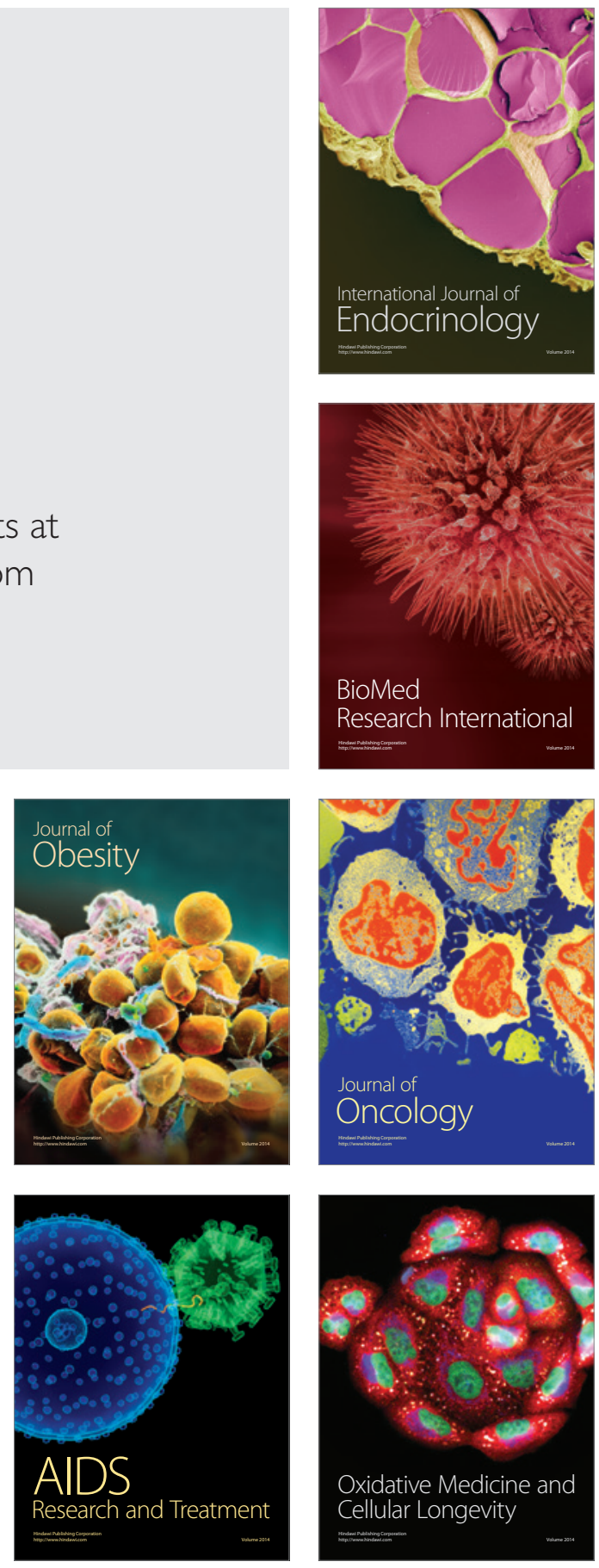